\title{
Revisión sistemática: El dobesilato de calcio mejora los síntomas de insuficiencia venosa de los miembros inferiores
}

Calcium Dobesilate for Chronic Venous Insufficiency: A Systematic Review. Ciapponi A;Laffaire E;Roque M. Angiology 2004;55(2):147-154.

\section{Objetivos}

Determinar la eficacia y la seguridad del dobesilato de calcio por vía oral en la insuficiencia venosa crónica (IVC).

\section{Fuente y selección de estudios}

MEDLINE, EMBASE y el Cochrane Controlled Trials Register (hasta Diciembre 2001), el registro de Laboratorios Esteve, listas de referencias de meta-análisis y artículos relacionados.Se incluyeron ensayos clínicos controlados, aleatorizados y doble ciego, cruzados o paralelos, de dobesilato contra otra alternativa terapéutica o placebo en la IVC, con un seguimiento mínimo de 28 días y con valoración de alguno de los siguientes resultados primarios (venas varicosas, edema, pigmentación cutánea, lipodermatoesclerosis, úlceras venosas, pesadez, calambres nocturnos, prurito, dolor) o secundarios (parámetros de pletismografía, doppler venoso, eco-Doppler, eco-doppler color y flebografía).

\section{Extracción de datos}

Dos revisores independientes, seleccionaron, valoraron la calidad de los estudios (mediante componentes individuales y escala de Jadad $^{*}$ y recolectaron los datos.

\section{Resultados}

De los diez ensayos identificados, seis cumplían con los criterios de inclusión y sólo dos eran de buena calidad metodológica (485 pacientes totales).

De los resultados disponibles evaluados el dobesilato de calcio, administrado por vía oral durante al menos cuatro semanas, mejoró en forma estadísticamente significativa los calambres nocturnos y las molestias ("disconfort"). Ver los resultados principales en la Tabla.

Tabla. Eficacia sintomática del dobesilato calcio en la IVC de los miembros inferiores.

\begin{tabular}{l|c|c}
\hline Sintomas & RR $^{*}$ de mejorí (IC 95\%) & NNT (IC95\%) \\
\hline Calambres nocturnos & $1,66(1,07-2,56)$ & $8(4-50)$ \\
\hline Molestias ("discomfort") & $2,30(1,51-3,52)$ & $4(3-7)$ \\
\hline
\end{tabular}

RR: riesgo relativo de dobesilato vs. Placebo;NNT:número necesario a tratar

Las mejorías fueron más marcadas en el subgrupo de pacientes con síntomas severos: Riesgo relativo (RR) de mejoría del dolor, la pesadez y el edema maleolar de 14 (IC95\% 4-53), 14 (IC95\% 2-93), y 27 (IC95\% 2-416) respectivamente; y NNTs cercanos a 1. No hubo diferencias significativas con el placebo en cuanto a la frecuencia de efectos adversos $(p=0,5)$. El mismo patrón se observó en la reducción del volumen de la pierna $(-1,6 \%$ entre los pacientes con síntomas leves y de $-7,2 \%$ entre los de síntomas severos). Usar una dosis de 1000 ó de $1500 \mathrm{mg} /$ día no modificó la magnitud de los resultados ni la tolerancia.

\section{Conclusiones}

Las evidencia disponible sugiere que el dobesilato de calcio es eficaz en la resolución de algunos síntomas de la insuficiencia venosa crónica. Existiría mayor eficacia en el subgrupo de pacientes de mayor gravedad; y la dosis de $1000 \mathrm{mg}$ /día podría tener comparable eficacia clínica y tolerancia que la de1500 mg/día.

Fuente de financiamiento: Laboratorios Doctor Esteve.

\section{Comentario}

La IVC de los miembros inferiores es una importante causa de incomodidad.Afecta al $10-15 \%$ de los varones y al $20-25 \%$ de las mujeres ${ }^{1}$.Se encuentra asociada a la historia de trauma de la pierna (Odds Ratio* $[O R]=2,4$ ) y a la de flebitis o trombosis venosa profunda $(O R=26)$. La incompetencia valvular secundaria a fenómenos post trombóticos es la más frecuente y representa el 80 al 95\% de los casos $^{2}$

Sus manifestaciones clínicas incluyen sensación de pesadez en las extremidades, parestesias, calambres, dolor, edemas, venas varicosas, pigmentación cutánea, várices dolorosas y signos de atrofia cutánea.

Los tratamientos farmacológicos son frecuentemente utilizados porque son fáciles de administrar y porque la adherencia a los tratamientos compresivos, el tratamiento de primera elección en ausencia de arteriopatía significativa, es pobre.

La mayoría de los flebotónicos son flavonoides naturales extraídos de las plantas. Hasta la publicación de este meta-análisis, existía evidencia de la efectividad de la "castaña de la India", que había demostrado reducir en forma significativa el dolor, el edema y el prurito asociado a la insuficiencia venosa en forma equivalente a la compresión graduada mediana ${ }^{3,1}$ y de los rutósidos, que habían mostrado alivio sintomático (dolor, calambres, "piernas cansadas" e hinchazón) sin existir diferencias clínicamente significativas entre ambos grupos ${ }^{4}$

Este impecable meta-análisis en el que participaron investigadores argentinos, uno de ellos miembro del comité editorial de nuestra revista, aporta nueva evidencia sobre la efectividad del dobesilato de calcio, especialmente en los pacientes con síntomas severos. En cuanto a su seguridad, que había sido cuestionada en un estudio de casos y controles ${ }^{5}$ por su asociación con agranulocitosis, podemos decir que por lo menos en el corto plazo, esta revisión no ha encontrado información alarmante. Sin embargo, como se trataría de eventos poco frecuentes habrá que estar atentos a los resultados de los programas de vigilancia epidemiológica.

Conclusiones del comentador: El dobesilato de calcio produce alivio de los síntomas de la insuficiencia venosa crónica, y es más eficaz en los pacientes con síntomas más severos.

* ver glosar io

\section{Dr. Sergio Terrasa [ Unidad de Medicina Familiar y Preventiva del Hospital Italiano de Buenos Aires. ]}

\footnotetext{
Referencias

1- Pittler MH, Ernst E.Horse chestnut seed extract for chronic venous insufficiency (Cochrane Review).In: The Cochrane Library, Issue 2, 2002. Oxford: Update Software.

2- Abidia A, Hardy SC. Surgery for deep venous incompetence (Cochrane Review). In: The Cochrane Library, Issue 2, 2002. Oxford: Update Software.

3- Diehm c, Trampisch H, Lange S, et. al.Comparisson of leg compression stokings and oral horse chestnut seed extract therapy in patients with chronic venous insufficiency.Lancet 1996 , 347 .292

4- Boada J N, Nazco G J. Therapeutic effect of venotonics in chronic venous insufficiency:a meta-analysis.Clinical Drug Investigation1999, 18(6), 413-432.

5- Ibañez L, Ballarín E, Vidal X, Laporte JR. Agranulocytosis associated with calcium dobesilate.Clinical course and risk estimation with the case-control and the case-population approaches. European Journal Clinical Pharmacology, 2000;56:763-7.
} 